\title{
Impact of reassessment of colonic hyperplastic polyps by expert GI pathologists
}

Schachschal, Guido ; Sehner, Susanne ; Choschzik, Matthias ; Aust, Daniela ; Brandl, Lydia ; Vieth, Michael ; Wegscheider, Karl ; Baretton, Gustavo B ; Kirchner, Thomas ; Sauter, Guido ; Rösch, Thomas

\begin{abstract}
BACKGROUND: Recommended follow-up intervals after endoscopic removal of hyperplastic polyps (HP) and sessile serrated adenomas (SSA) differ because of assumed differences in biological behaviour. However, histopathologic differentiation is difficult, with higher SSA rates reported from specialist GI histopathologists. OBJECTIVE: The objective of this study was to clarify the relevance of histologic reassessment of HP. DESIGN AND SETTING: From a prospective screening colonoscopy study relevant serrated lesions (excluding distal small HP $5 \mathrm{~mm}$ ) diagnosed by private practice pathologists were reassessed by four specialized GI pathologists PATIENTS: One thousand sixty-nine screening colonoscopies were performed in patients. MAIN OUTCOME MEASUREMENTS: In terms of main outcome measurements, there is a likelihood of changes of the HP diagnosis on reassessment, as well as interrater variability. RESULTS: SSA were initially diagnosed in 7 cases $(0.7 \%)$ and relevant HP in 83 (7.8\%; 101 lesions). Of the latter, the chance of a change in diagnosis from HP to SSA by any of the four specialist histopathologists was higher for larger $(>5 \mathrm{~mm})$ and right-sided lesions (19.1 vs $1.3 \%$, OR 18.4, $\mathrm{p}=0.04)$ including a higher likelihood to change recommended follow-up intervals $(32.1 \mathrm{vs} 3.3 \%$, $\mathrm{p}<0.01)$. However, follow-up intervals were determined by concomitant adenomas in $41 \%$. Interrater variability was also higher for these lesions $(\mathrm{p}=0.04)$, with an overall kappa value of 0.48 . However, this issue related to only $1.2 \%$ of the 1069 study cases. LIMITATION: The limitations this study are the limited case number as well as limited retrospective assessment. CONCLUSIONS: Right-sided HP >5 mm had a higher chance of change in diagnosis to SSA; therefore, they should probably be treated like adenomas and be removed. However, reliable data for recommendations on follow-up intervals of HP or SSA will require follow-up studies.
\end{abstract}

DOI: https://doi.org/10.1007/s00384-016-2523-8

Posted at the Zurich Open Repository and Archive, University of Zurich

ZORA URL: https://doi.org/10.5167/uzh-124034

Journal Article

Accepted Version

Originally published at:

Schachschal, Guido; Sehner, Susanne; Choschzik, Matthias; Aust, Daniela; Brandl, Lydia; Vieth, Michael; Wegscheider, Karl; Baretton, Gustavo B; Kirchner, Thomas; Sauter, Guido; Rösch, Thomas (2016). Impact of reassessment of colonic hyperplastic polyps by expert GI pathologists. International journal of colorectal disease, 31(3):675-683.

DOI: https://doi.org/10.1007/s00384-016-2523-8 


\title{
Impact of reassessment of colonic hyperplastic polyps by expert GI pathologists
}

\author{
Guido Schachschal ${ }^{1}$ - Susanne Sehner ${ }^{2} \cdot$ Matthias Choschzick $^{3,4}$ • Daniela Aust ${ }^{5}$ • \\ Lydia Brandl $^{6}$ - Michael Vieth ${ }^{7} \cdot$ Karl Wegscheider $^{2}$ - Gustavo B. Baretton ${ }^{5}$. \\ Thomas Kirchner ${ }^{6} \cdot$ Guido Sauter $^{3} \cdot$ Thomas Rösch $^{1}$
}

Accepted: 27 January 2016 /Published online: 4 February 2016

(C) Springer-Verlag Berlin Heidelberg 2016

\begin{abstract}
Background Recommended follow-up intervals after endoscopic removal of hyperplastic polyps (HP) and sessile serrated adenomas (SSA) differ because of assumed differences in biological behaviour. However, histopathologic differentiation is difficult, with higher SSA rates reported from specialist GI histopathologists.

Objective The objective of this study was to clarify the relevance of histologic reassessment of HP.

Design and setting From a prospective screening colonoscopy study relevant serrated lesions (excluding distal small HP $\leq 5 \mathrm{~mm}$ ) diagnosed by private practice pathologists were reassessed by four specialized GI pathologists

Patients One thousand sixty-nine screening colonoscopies were performed in patients.
\end{abstract}

Thomas Rösch

t.roesch@uke.de

1 Department of Interdisciplinary Endoscopy, University Hospital Hamburg-Eppendorf, Martinistr 52, 20246 Hamburg, Germany

2 Department of Medical Biometry and Epidemiology, University Hospital Hamburg-Eppendorf, Hamburg, Germany

3 Department of Pathology, University Hospital Hamburg-Eppendorf, Hamburg, Germany

4 Institute of Surgical Pathology, University Hospital Zurich, Zurich, Switzerland

5 Department of Pathology, University Hospital Carl Gustav Carus, Dresden, Germany

6 Institute of Pathology, Ludwig-Maximilian University, Munich, Germany

7 Institute of Pathology, Bayreuth Hospital, Bayreuth, Germany
Main outcome measurements In terms of main outcome measurements, there is a likelihood of changes of the HP diagnosis on reassessment, as well as interrater variability.

Results SSA were initially diagnosed in 7 cases $(0.7 \%)$ and relevant HP in 83 (7.8\%; 101 lesions). Of the latter, the chance of a change in diagnosis from HP to SSA by any of the four specialist histopathologists was higher for larger ( $>5 \mathrm{~mm}$ ) and right-sided lesions (19.1 vs $1.3 \%$, OR 18.4, $p=0.04$ ) including a higher likelihood to change recommended follow-up intervals ( 32.1 vs $3.3 \%, p<0.01)$. However, follow-up intervals were determined by concomitant adenomas in $41 \%$. Interrater variability was also higher for these lesions $(p=0.04)$, with an overall kappa value of 0.48 . However, this issue related to only $1.2 \%$ of the 1069 study cases.

Limitation The limitations this study are the limited case number as well as limited retrospective assessment.

Conclusions Right-sided HP $>5 \mathrm{~mm}$ had a higher chance of change in diagnosis to SSA; therefore, they should probably be treated like adenomas and be removed. However, reliable data for recommendations on follow-up intervals of HP or SSA will require follow-up studies.

Keywords Histopathological second opinion - Serrated lesions $\cdot$ Hyperplastic polyps $\cdot$ Screening colonoscopy

\section{Introduction}

Colorectal polyps are increasingly detected during colonoscopy both due to more colonoscopies being performed within various screening programmes and due to increasing awareness of endoscopists; recently, it has been shown more adenomas are found during screening colonoscopy within the German screening programme, just to name one example 
[1]. Therefore, the correct recognition, assessment and classification of colorectal polyps are of paramount importance for further management. Of these polyps, serrated polyps/lesions or adenomas have recently attracted much attention [2].

Serrated colonic lesions consist of several subgroups, the most important of which are hyperplastic polyps (HP) and sessile serrated adenomas (SSA) in terms of frequency. The biological behaviour of those two types is considered to be different: SSA are widely recognized as precursor lesions of serrated pathway colorectal carcinoma [2, 3], while HP are still considered to have no malignant potential. The prevalence of SSA remains unclear, varying between $2.8 \%$ (standard care) and $9 \%$ (single-expert endoscopy and pathology reports) in patients undergoing screening and/or diagnostic colonoscopy [4-7]. Similarly, although there are two different variants of the pathway from serrated adenomas to colorectal carcinoma with regards to cancer aggressiveness [8], the relevance in clinical practice especially with regards to screening colonoscopy and associated adenoma findings is not yet clear.

A detection rate of $5 \%$ has been suggested for proximal serrated lesions for average-risk men and women undergoing screening colonoscopy [9] including both HP and SSA. With regards to their relative distribution within the group of serrated lesions, the rise of SSA detection rate over time [10] could be at least partially due to a shift in diagnosis from hyperplastic polyps on histopathological review [11, 12]. The distinction between SSA and HP, however, may be difficult, and previous studies have also reported poor to fair interobserver agreement rates for histopathological diagnosis of SSA versus HP, with kappa values ranging from 0.16 to $0.38[11,13,14]$. For these reasons, diagnostic criteria for SSA have been recently refined by several expert panels and consensus conferences $[2,15]$, in the hope of arriving at a more uniform and reproducible histopathological diagnosis.

From a clinical point of view, guideline follow-up intervals differ for true hyperplastic polyps (if they are the only polyps found) and for SSA. The recommended follow-up for hyperplastic polyps is 10 years, whereas that for SSA, which is analogous with those of conventional adenomas, varies between 3 and 5-10 years, depending on size, number and histology $[16,17]$. Because of the uncertainties in histological diagnosis mentioned above, the current study analyses the value of a second opinion for smaller serrated polyps $(\leq 10 \mathrm{~mm})$ removed during screening colonoscopy.

\section{Methods}

\section{Case selection and reassessment}

All colonic hyperplastic polyps were selected from a previously published study [18] on the endoscopic differential diagnosis of colonic polyps, which had been originally interpreted as hyperplastic polyps by private practice pathologists. Out of these, hyperplastic polyps that were distally located (below the splenic flexure) and were $\leq 5 \mathrm{~mm}$ in size were excluded: This was done because such small whitish polyps can usually be recognized at endoscopy as hyperplastic and are almost always histologically confirmed as such if resected or removed under biopsy. Furthermore, for these reasons, they are often even left in place especially if they are diminutive and multiple. Thus, in summary, the case selection for this study included all HP on the right side (oral of the splenic flexure) and HP $>5 \mathrm{~mm}$ on the left side (aboral of the splenic flexure).

These selected cases were pseudonymized (only sex and age were known) and were reassessed by four specialized gastrointenstinal (GI) pathologists (M.C., D.A., L.B., M.V.) using the original haematoxylin and eosin $(\mathrm{H} \& \mathrm{E})$-stained slides with serial sections; the diagnostic criteria for SSAs are shown in Table 1. These histopathologists had specific interest in colonic pathology and had already published on the topic and/or were involved in consensus meetings on polyps and SSA. To mimic a second-opinion procedure, the four pathologists were not blinded to the original diagnosis by community pathologists (namely, all diagnosed as HP) of the lesions they reviewed. That is, the histopathologists involved in reassessment knew that they reassessed polyps of all sizes in the right colon and polyps of $5 \mathrm{~mm}$ and more in the left colon which had been originally given the diagnosis of a hyperplastic polyp in the primary histopathological workup; this closely mimics a second-opinion process. In their reassessment, they had to choose between genuine HP, SSA, traditional serrated adenoma (TSA) and conventional adenoma. As required by the German reimbursement system, at least eight serial sections have to be available per polyp; all histopathologists received these serial sections for reanalysis.

With regards to removal technique, all polyps of more than $5 \mathrm{~mm}$ diameter had been removed by snare, and smaller polyps by either snare of biopsy forceps using multiple bites.

Table 1 Microscopic criteria for diagnosis of SSA according to the German Society of Pathology [15]

\footnotetext{
- Hyperserration, serration in the lower third of the crypts with and without branching of the crypts

- T- or L-shaped crypts above the muscularis mucosae

- Inverted crypts (pseudoinvasion) below the muscularis mucosae

- Columnar dilatation in the lower third of the crypts (with and without presence of mucus)

- Shifting of proliferation zone to the middle third of the crypts

- Vesicular nuclei with nucleoli

- Mature goblet cells at the base of the crypts
} 


\section{Outcomes}

Main outcome There is likelihood of a change in diagnosis (from HP to SSA or adenoma) on a lesion basis.

Secondary outcomes • There is a likelihood of a shortening of recommended follow-up intervals on a per-patient basis, namely, shortening from 10 years (based on a diagnosis of HP only) to either 3 or 5-10 years based on the presence of adenomas. For this analysis, SSA were treated like adenomas with respect to follow-up recommendations [16, 17], although this practice might not be regarded as finally established. Follow-up recommendations were based on recent guidelines which can be summarized for this analysis as follows: 5-10 years are recommended for one to two adenomas $<10 \mathrm{~mm}$ in size, while 3 years are recommended for three to ten adenomas even if smaller (the other criteria relate to larger sizes or more advanced histology than that found in our study on small polyps) $[16,17]$. To categorize follow-up intervals, 3 and 510-year recommendations were taken together as "shortening in follow-up intervals" due to limited sample size of HPs selected for the study.

- There is an interrater variability between the four specialized GI pathologists in the diagnosis of HP versus adeno$\mathrm{ma} / \mathrm{SSA}$.

\section{Statistical analysis}

Change in diagnosis and subsequent change in follow-up recommendation For these analyses, change of diagnosis of polyps was considered on a lesion basis, while change in follow-up recommendation was analysed on a per-patient basis (for patients without concomitant adenomas). We used the following assumptions: A "change in diagnosis/ follow-up" would occur if any histopathological second opinion led to the diagnosis "not HP but adenoma and/or SSA". As predictors for "change in diagnosis/follow-up yes/no", we used the following variables: size, location (left/right), interaction between size and location, age, sex and occurrence of one or more lesion. Additionally, we employed the same model but using size as a categorical variable with the cutoff of $5 \mathrm{~mm}(\leq 5 \mathrm{vs}>5 \mathrm{~mm})$.

We estimated a random intercept model with change in follow up yes/no as outcome and crossed random effects of pathologist and patient. The benefit of this modelling is that both pathologist and patient were treated as random selections. For both, the median odds ratios (MORs) were applied to quantify the variation between clusters by comparing two different patients (or two pathologists) from two randomly chosen, different clusters, as outlined in detail elsewhere [19]. Principally, as described in that paper, when two persons with the same covariates, chosen randomly from two different clusters, are considered, "The MOR is the MOR between the person of higher propensity and the person of lower propensity. ... The measure is always greater than or equal to 1 . If the MOR is 1 , there is no variation between clusters (no second-level variation). If there is considerable between-cluster variation, the MOR will be large. The measure is directly comparable with fixed-effects odds ratios".

Analogous modelling was used for estimating the likelihood of a change in diagnosis on a lesion basis. The random intercept was changed from patient to lesion clusters, and an additional predictor, namely, coexistence of adenoma(s) in the same patient, was included in the model. Using the likelihood ratio test, all insignificant interactions and variables were excluded from the models (backward elimination).

Interobserver agreement We fitted an ordinal regression with the following agreement outcomes: $0=$ no assessment possible; 1 =two out of four pathologists agreed; 2 =pairwise agreement; $3=$ three out of four pathologists agreed; and $6=$ total agreement. As predictors for agreement, we used the following variables: size, location (left/right), interaction between size and location, age, sex, occurrence of one or more lesion(s) and coexistence of adenoma(s) in the same patient.

Nominal $p$ values are reported without correction for multiplicity, and $p$ values $<0.05$, two-sided, were considered significant. All analyses were conducted using Stata 13.1 (StataCorp, College Station, Texas, USA.

\section{Results}

\section{Patients and polyps}

Patient numbers for polyps of different histology and the case selection for this study are shown in Fig. 1. The adenoma detection rate, that is, the percentage of patients with at least one adenoma detected, was $28.2 \%$, and 170 
Fig. 1 Flowchart showing study patients, group distribution with regard to polyp type, result of histopathological reassessment of polyps and change in follow-up recommendations (see text). SSA sessile serrated adenomas.

$*$ Excluded from reassessment since HP size was $<5 \mathrm{~mm}$. **See Fig. 2 for results of reassessment

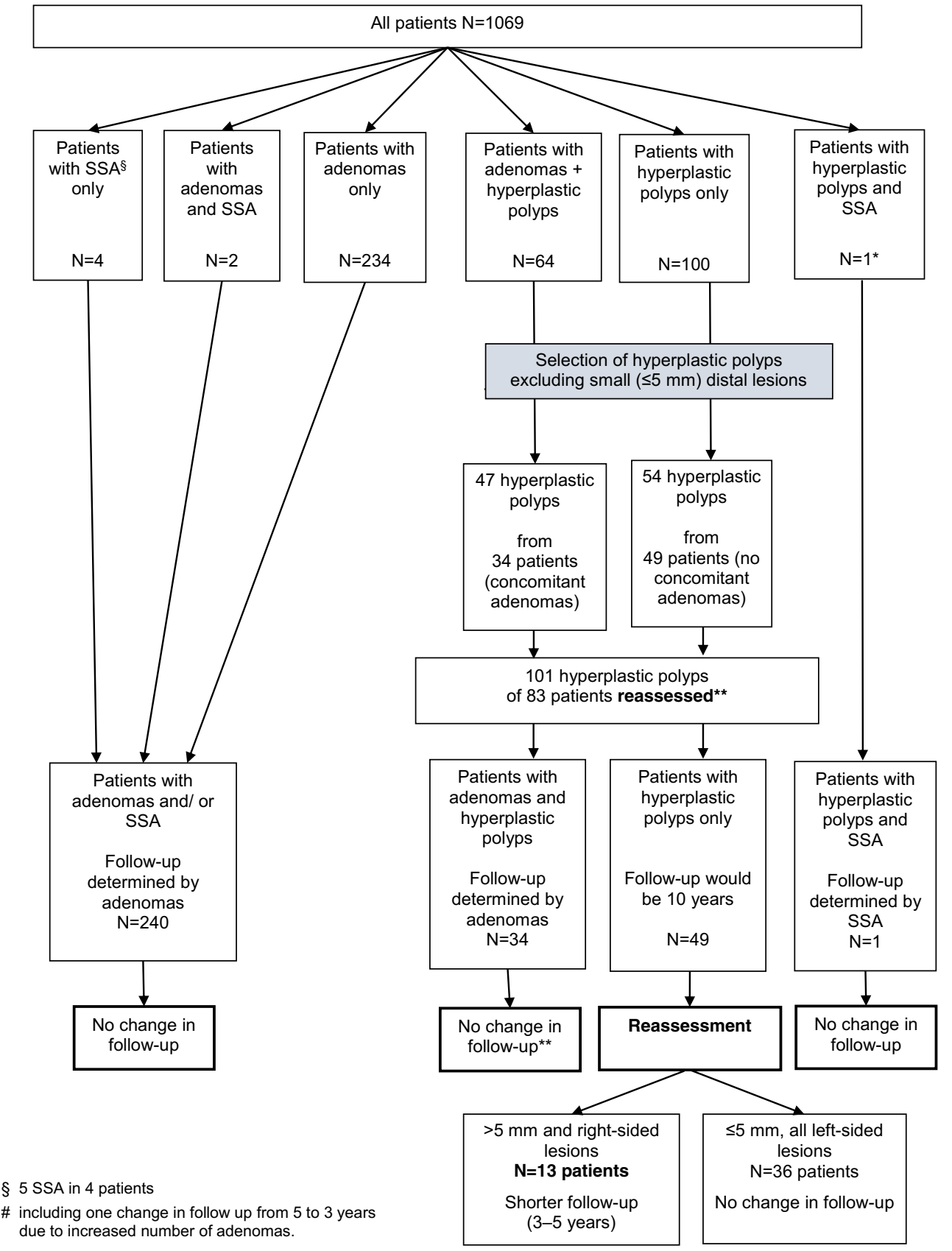

patients were found to have serrated lesions, i.e., HP and SSA $(170 / 1069 ; 15.9 \%)$. Amongst these 170 patients, there were 101 hyperplastic polyps that met the inclusion criteria for this study, found in 83 patients (41 women, 42 men; mean age 62 years, range 52-84 years). In addition, six further serrated lesions had been primarily diagnosed as SSA by community pathologists but were not included in the reassessment.

The mean polyp size was $5 \mathrm{~mm}$ (range 1-10 mm); $34 \mathrm{HP}$ were left-sided with mean size of $6 \mathrm{~mm}$ (size range $5-10 \mathrm{~mm}$, see inclusion criteria), and 67 were right-sided with mean size $4 \mathrm{~mm}$. No HP larger than $10 \mathrm{~mm}$ was found during the study in the 1069 patients. Biopsy forceps had been used to remove 51 lesions and snare polypectomy for 50 lesions.

\section{HP polyp reassessment (second opinion, 101 lesions in 83 patients)}

Figures 1 and 2 show the results of the reassessment of the 101 hyperplastic polyps by four specialized GI pathologists.

Likelihood of a change in diagnosis The likelihood for a change in diagnosis from HP to SSA by any of the four specialized histopathologists was higher for right-sided, 


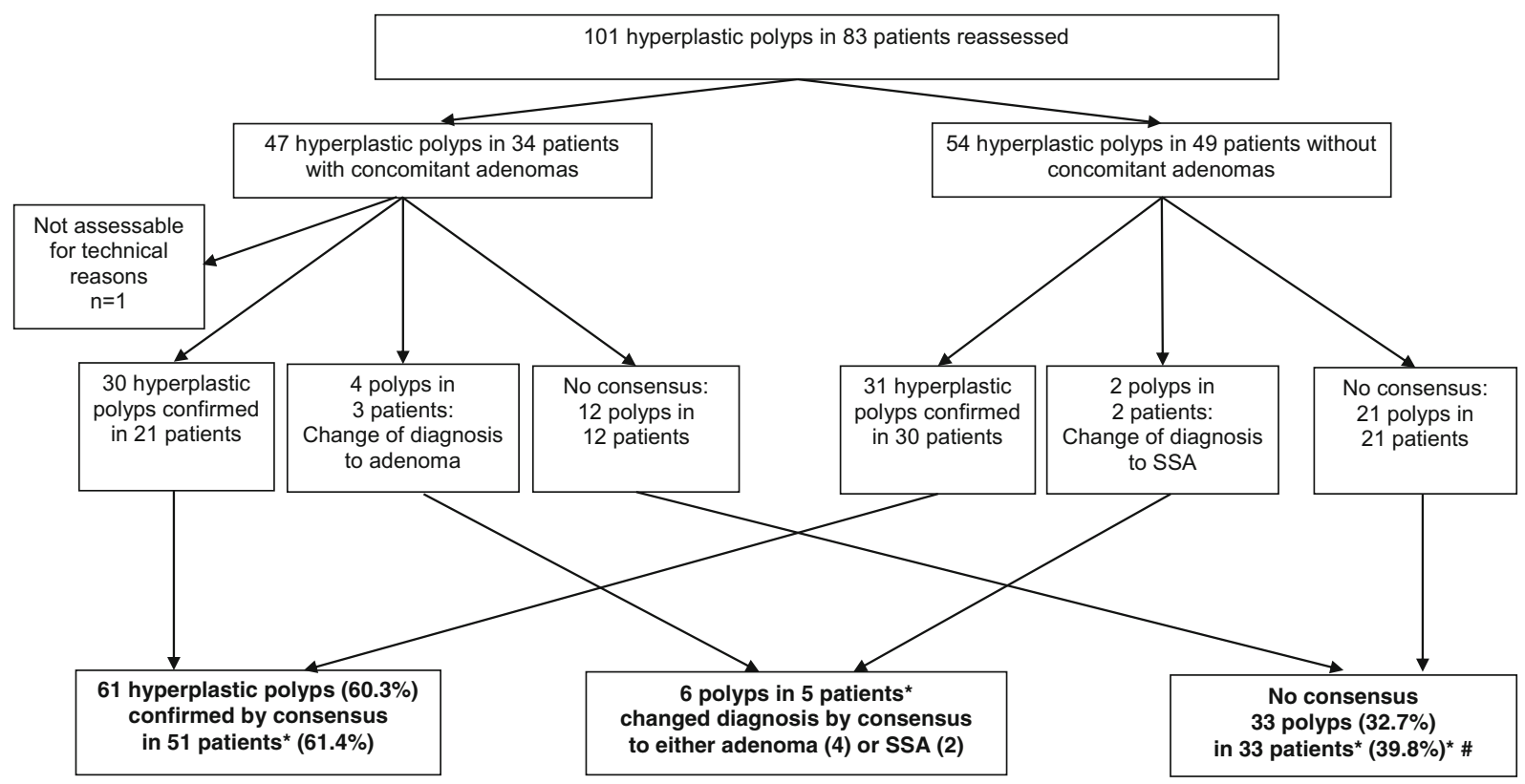

*The total number of polyps is $101(61+6+33+1)$, but the total number of patients is higher $(51+5+33=89$ instead of 83$)$ since there were 6 patients with at least two hyperplastic polyps who appear in two different subgroups with regard to consensus.

Fig. 2 Flowchart showing reassessment results (see text). *The total number of polyps is $101(61+6+33+1)$, but the total number of patients is higher $(51+5+33=89$ instead of 83$)$ since there were six

larger ( $>5 \mathrm{~mm}) \mathrm{HP}$ lesions, at 19.1 versus $1.3 \%$, with an OR of 18.4 (95\% CI 1.1-294, $p=0.04)$. None of the other analysed factors showed any effect. Endoscopic and histologic examples are shown in Figs. 3 and 4.

\section{Interobserver agreement (Table 2)}

Overall kappa for the confirmation of HP was 0.448 (95\% CI $0.28-0.60 ; p<0.001)$. Using ordinal regression for the degree of agreement defined above, after the backward elimination, only the interaction between size and location and the patient age showed a significant influence on interobserver agreement. The proportional odds assumption was not violated.

With increasing patient age, the probability of agreement decreased (OR 0.92, $95 \%$ CI 0.86-0.99; $p=0.024$ ). For leftsided lesions, polyp size had no effect on agreement, with an OR of 1.01 (95\% CI 0.58-1.76; $p=0.967)$. Over all observed sizes $(5-10 \mathrm{~mm})$ for left-sided lesions, the adjusted probability of total agreement was $72.5 \%$ (95\% CI 55.4-89.7\%). For right-sided lesions, the probability of agreement decreased by size, with an OR of 0.55 (95\% CI $0.42-0.73 ; p<0.001)$. This different effect of size by location was significant $(p=0.039)$. In summary, agreement was worst for larger $(>5 \mathrm{~mm})$ rightsided lesions (OR 0.22, $95 \%$ CI $0.07-0.65, p=0.006$ ) as compared to smaller and left-sided polyps. This is shown in detail in Fig. 5. patients with at least two hyperplastic polyps who appear in two different subgroups with regard to consensus
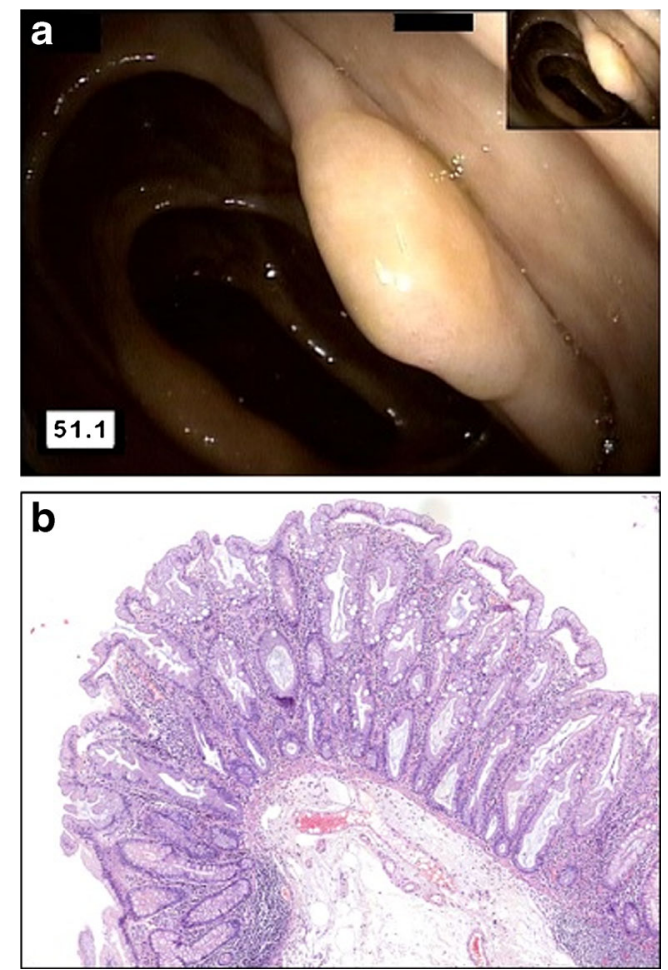

Fig. 3 Ten millimeter-right-sided flat lesion, with indeterminate assessment by the four GI histopathologists (two hyperplastic/two sessile serrated adenoma). a Endoscopy, very flat lesion with glossy appearance from some distance, considered typical for sessile serrated adenoma. b Histology, a hyperplastic polyp with serration, no complex architecture, no micro-herniation, no basal goblet cells and no T-shaped or L-shaped glands 
Fig. 4 Five millimeter-left sided sharply demarcated polyp (reassessment 3:1 for sessile serrated adenoma vs hyperplastic polyp). a Endoscopy (white light, left, and i-scan mode, right) shows a sessile lesion with villous features suggesting adenoma.

b Histology shows a polyp with serration, tangential cut mimicking micro-herniation, but no complex architecture, no microherniation and some basal goblet cells, T- or L-shaped glands seem to be present but, due to tangential cut, hard to assess. As far as from this tangential cut, it could be concluded that the lesion is representing a hyperplastic polyp

\section{a}

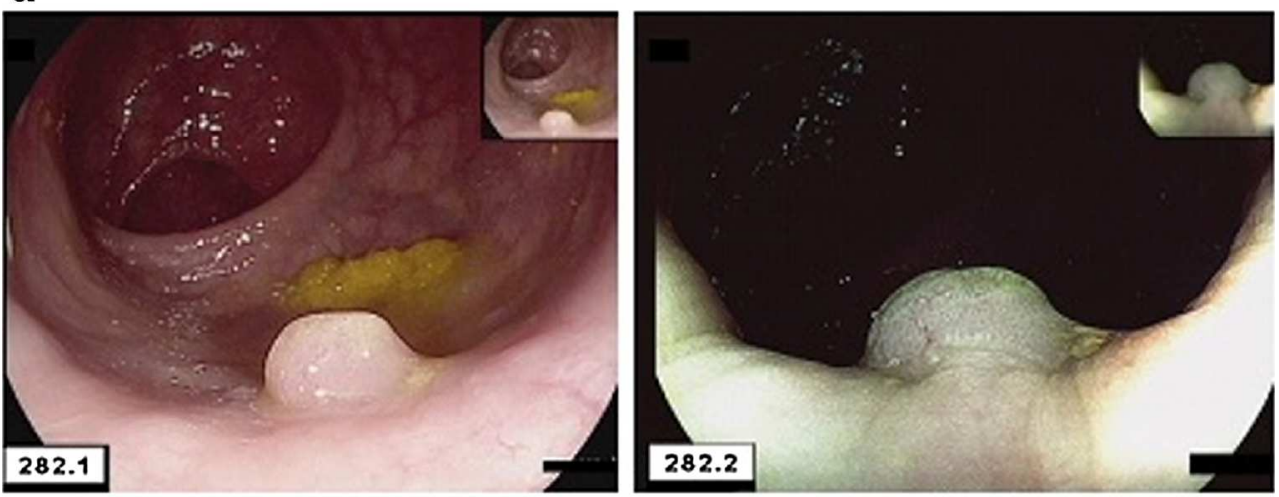

b

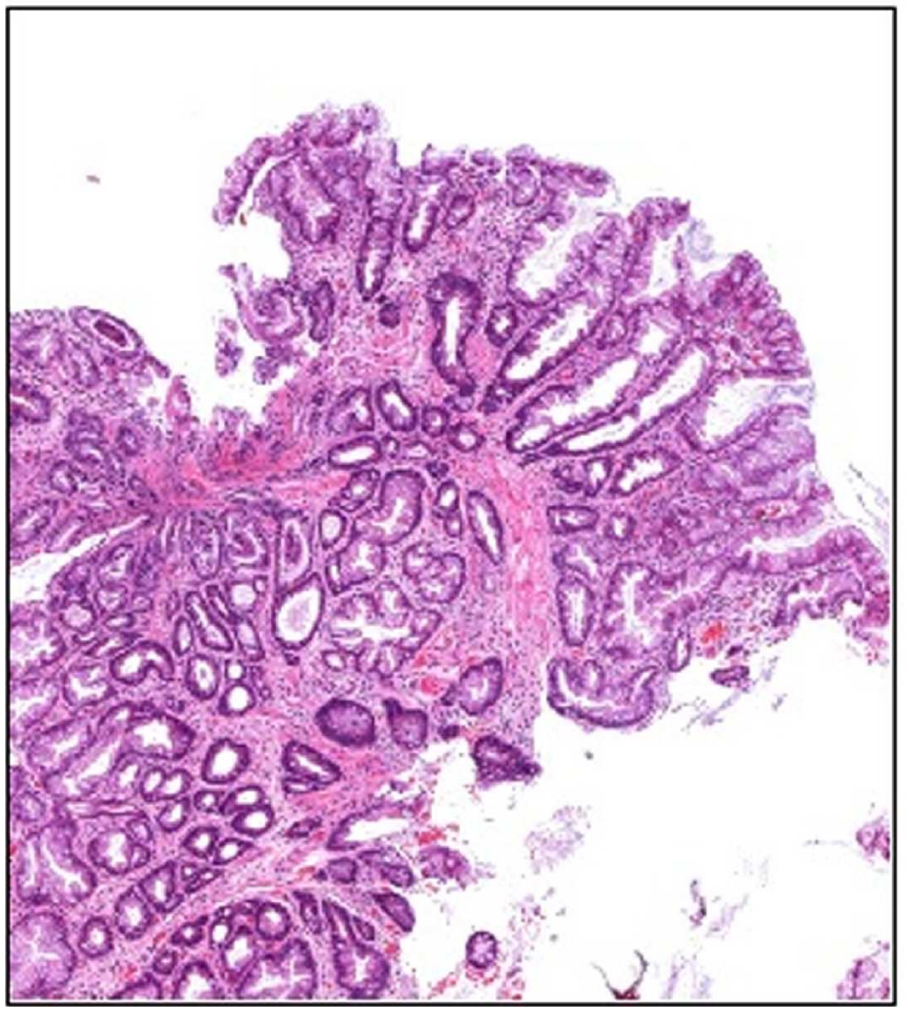

\section{Change in follow-up recommendations}

In patient group, follow-up would change to shorter intervals with a change in diagnosis to SSA (or adenoma) after

Table 2 Kappa values for four raters in relation to the histological diagnosis

\begin{tabular}{llll}
\hline Outcome & Kappa & $\mathrm{N}$ & $p$ Value \\
\hline Not assessable & 1 & 1 & $<0.001$ \\
HP or less & 0.4696 & 61 & $<0.001$ \\
Adenoma & 0.8493 & 4 & $<0.001$ \\
SSA & 0.3123 & 2 & $<0.001$ \\
Combined & 0.4838 & & $<0.001$ \\
\hline
\end{tabular}

reassessment of HP. These possible changes in follow-up recommendations based on a change in HP diagnosis following histopathological reassessment are shown in Fig. 1. Overall, 34/83 patients (41\%) had concomitant adenomas/ SSA; these determined the follow-up interval, and these patients are not considered in the following analyses. The subgroup of HP patients without concomitant adenomas/ SSA comprised 49 patients ( 28 women, 21 men; mean age 63 years, range 52-84) with 54 lesions. Mean polyp size was $5.22 \mathrm{~mm}$ (range $2-10 \mathrm{~mm}$ ); 17 lesions were in the left colon ( 3 lesions $>5 \mathrm{~mm}, 17.7 \%$ ), and 37 were right-sided (14 lesions $>5 \mathrm{~mm}, 37.8 \%$ ). In only one case, the additional diagnosis of a small adenoma from the original diagnosis of HP would have shortened follow-up intervals due to the increase in numbers of adenomas from two to 
Fig. 5 Estimated effect of lesion location and size on interrater agreement, according to left-sided (blue) and right-sided (green) polyp location

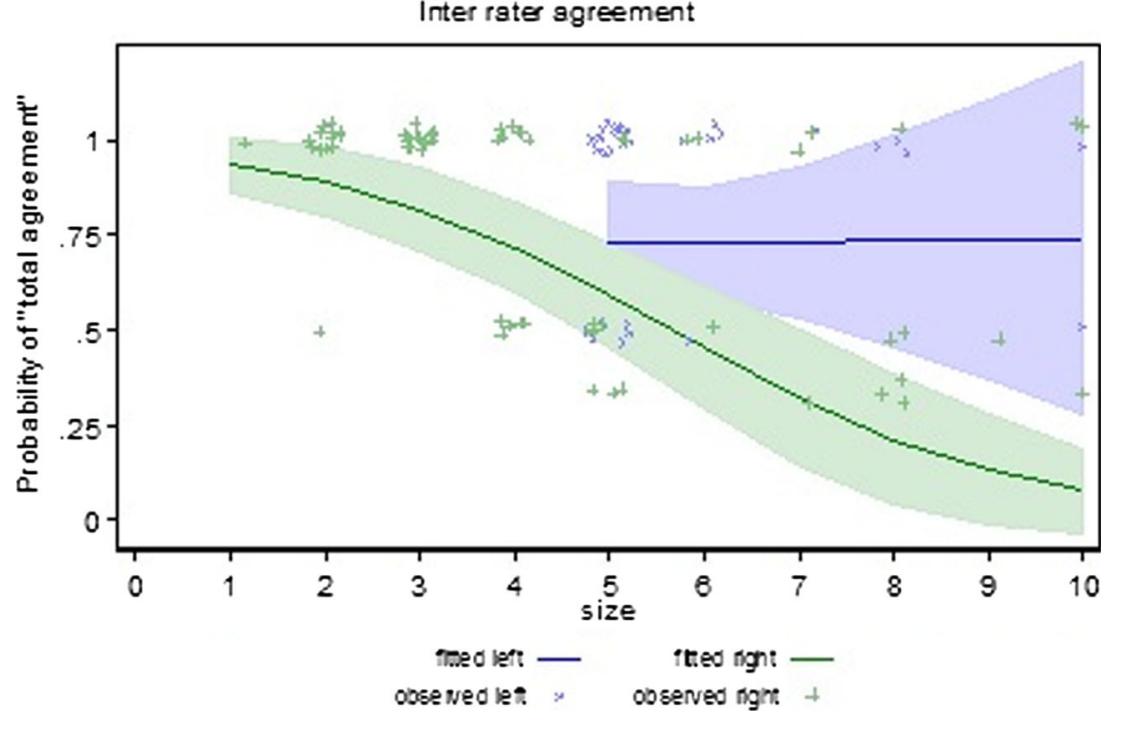

three; the other patients had less than three adenomas even with diagnostic changes after reassessment.

Overall, also in this subgroup, the likelihood of reduction of follow-up interval was also significantly increased for patients with larger $(>5 \mathrm{~mm})$ right-sided lesions $(32.1 \%, 95 \%$ CI $0-71.2 \%$ vs $3.3 \%, 95 \%$ CI $0-9.2 \%$; $p<0.01$ ), resulting in an almost 14-fold higher chance as compared with patients with all other lesions $(\mathrm{OR}=13.8,95 \%$ CI 2.3-84.7; $p=0.004)$. With regards to the influence of different pathologists and possible differences between patients, the MOR was $3.2(95 \%$ CI 1.6-15.8) for pathologists and 6.2 (95 \% CI 2.8 25.2) for patients (see "Methods" section). In relation to the entire patient group $(n=1069)$, changes in follow-up recommendation arising from histopathological reassessment of HP would occur in only about $1 \%$ of cases overall (see Fig. 1).

\section{Discussion}

Colorectal adenomas are known precursor lesions of colorectal carcinoma, and on the detection and removal of these adenomas rests the preventive effect of colorectal cancer screening including stool tests and colonoscopy. Up to now, these polyps were mainly differentiated into hyperplastic polyps and adenomas. In recent years, sessile and TSAs have been identified as separate lesions which may be precursor lesion in the so-called serrated and alternate pathways of colorectal carcinogenesis $[3,20]$. Therefore, correct diagnosis and differential diagnosis of serrated lesions (hyperplastic polyps, sessile and TSAs) is very important for further management including follow-up.

Among colonic serrated lesions, SSA have aroused great interest in recent years, mainly because of their different pathway to colorectal cancer [21, 22]. They could also be missed more frequently, since they are mostly flat lesions and located in the right colon. Histological criteria have been developed on the basis of morphological changes and marker expressions $[2,15]$. The malignant potential of SSA has been regarded as substantial by some groups [23], but no consensus exists about both their occurrence and overall cancer risk in a broad community setting. "Truly" hyperplastic polyps constitute separate subgroups in the WHO classification of serrated lesions in the colorectum [24]; in the past, they have been considered to harbour no malignant potential [25]. This, however, seems to be based on morphological assessment rather than on large and meticulous follow-up studies; furthermore, recent data suggest that mutational changes may already have occurred at the hyperplastic polyp stage [26].

With regard to the histopathological diagnosis of serrated lesions, it has been shown previously that the diagnosis of these polyps made by community histopathologists is often not confirmed in a second-opinion procedure by specialized GI pathologists - findings confirmed by our study. In earlier studies on serrated lesions, it has already been stated that "there is great morphologic variability in SPs [serrated polyps] in the large intestine and what has been considered by some to be a morphologic continuum between HPs and SSA (e.g., as part of the "hyperplastic polyp-serrated adenoma-carcinoma sequence") may not truly be a continuum but rather may represent an inability to separate these lesions based on a superficial evaluation of the morphologic features" [24]. In 2009, Douglas Rex's group showed that in a historical group of 40 hyperplastic polyps diagnosed in $2001,30-85 \%$ of these polyps were reinterpreted as SSA by gastrointestinal pathologists in 2007. Already then, interobserver agreement between specialized GI pathologists was seen to be poor with a very low kappa value (0.16) [11]. Our kappa value of 0.48 is in the moderate range, in line with most interobserver results in histopathologic and endoscopic assessment studies, especially when observers from different departments are involved, 
which much more relates to daily reality than experts from one single center.

The low rate of SSA diagnosed primarily in our study may reflect a lower rate during screening in daily practice as well as an underdiagnosis by community pathologists; taken together with HP, the overall rate of serrated lesions $(8.4 \%)$ correlates well with other papers: A study from Canada in 2012 demonstrated that in a provincial pathology database including 4096 colon polyps, 635 were reported as hyperplastic, most of them $(81.3 \%)$ being left-sided. Of the 119 right-sided HP, 114 were re-analysed by two specialized GI pathologists using a mutual consensus process, alongside 90 randomly selected left-sided HP. In this study, $17 \%$ of right-sided polyps and $20 \%$ of those $>5 \mathrm{~mm}$ were reclassified as SSA [12]. In a similar study from Australia, 6340 colorectal polyps diagnosed by one histopathologist were reviewed with regards to the occurrence of SSA which formed $12.1 \%$ of this group $(n=741) ; 80 \%$ were located in the proximal colon. Interobserver concordance was analysed in a small subset of cases $(n=90)$ and was found to be good (kappa 0.66). Of the SSA subtypes, concordance was $100 \%$ for SSA and $90 \%$ for pSSA, defined as below the WHO definition threshold for SSA-type crypt number [27]. In contrast, two further studies on interobserver agreement found rather disappointing results $[13,14]$.

Our study was different from previous ones as we focused on serrated polyps up to $10 \mathrm{~mm}$ (none was larger than $10 \mathrm{~mm}$ in our patient group) and excluded obvious and "easy" HP cases, namely, diminutive polyps $(<5 \mathrm{~mm})$ in the distal colorectum. Thus, the subgroup represents the most difficult cases for histopathological assessment; on the other hand, these smaller lesions are those for which the histopathological classification is discussed most. In our study with an overall adenoma detection rate of $28 \%$, the rate of sessile serrated lesions in the entire patient group was $15.9 \%$ and the rate of proximal serrated lesions $5.4 \%$. Our private practice histopathologists may not be comparable with community pathologists included in other studies since they represent variable levels of experience and specialization, but none of them with specific and scientific interest in colonic serrated adenomas.

We also attempted to bring together the consequences of a second-opinion process with the issue of interobserver agreement: Although histopathology re-review of polyps diagnosed primarily as hyperplastic revealed different diagnoses from HP in $40 \%$ of cases, interobserver agreement among the GI pathology specialists was moderate. Even more importantly, however, proximal and larger HP lesions had a high risk of a change in diagnosis but also had the highest interrater variability among GI specialized histopathologists. These results reflect the different weighting of diagnostic criteria for serrated lesions by the pathological community. Thus, a second opinion process is not worthwile at the present state of knowledge in daily practice given the uncertainties in endoscopic and histopathologic diagnosis and the lack of knowledge of long-term behaviour of serrated lesions. In the meantime, and in the light of our results and those of others, we would recommend to remove these lesions as if they were adenomas and not remove them under biopsy.

New and cost-effective histopathological markers are therefore in great demand, at least theoretically. It is likely that using markers such as BRAF, c-MYC oncogene and SIRT1 protein may be helpful in determining the risk status of serrated lesions [23] but may significantly increase costs of histopathological analysis. As mentioned, it is still unclear, whether, alternatively, sophisticated endoscopic methods may provide similar results in the future. Studies on follow-up after removal of sessile lesions are required to see whether there are any differences between them beyond the well known parameters of patient age and sex and polyp size, form and location.

For the time being, it could be considered whether rightsided HP lesions $>5 \mathrm{~mm}$ should receive a follow-up regimen similar to that of SSA and adenomas, even if the primary histopathologic diagnosis was hyperplastic polyp. We could show that histological reassessment within a second-opinion process does not make sense, since there was both a high likelihood for a change in diagnosis and at the same time a high interrater variability. Finally, this would concern only a small minority of patients $(1 \%$ overall and $6 \%$ of the 83 patients with HP only) as also shown in our study, since about half of HP patients have concomitant adenomas which almost always determine follow-up intervals.

Contribution of authors Guido Schachschal was responsible for designing and conducting the analysis, data collection and writing of the paper; Matthias Choschzcik, Daniela Aust, Lydia Brand and Michael Vieth did the histopathological reassessment; finally Susanne Sehner and Karl Wegscheider carried out the statistical analysis. Gustavo Baretton, Thomas Kirchner and Guido Sauter were involved in advising on the classification system and especially in paper revision. Daniel von Renteln, Guido Schachschal and Tania Noder contributed to data collection. Jan Kersten was responsible for the statistical analysis. Thomas Rösch was responsible for designing and conducting the study, data collection, data analysis and writing and revising of the manuscript. All authors revised and approved the final manuscript.

\section{Compliance with ethical standards}

Conflict of interest All authors state that they have no conflict of interest or financial ties relevant to this study to disclose.

\section{References}

1. Brenner H, Altenhofen L, Kretschmann J, et al. Trends in adenoma detection rates during the first $10 \mathrm{y}$ of the German Screening Colonoscopy Program. Gastroenterology 2015

2. Rex DK, Ahnen DJ, Baron JA et al (2012) Serrated lesions of the colorectum: review and recommendations from an expert panel. Am J Gastroenterol 107:1315-1329, quiz 1314, 1330 
3. Rosty C, Hewett DG, Brown IS et al (2013) Serrated polyps of the large intestine: current understanding of diagnosis, pathogenesis, and clinical management. J Gastroenterol 48:287-302

4. Abdeljawad K, Vemulapalli KC, Kahi CJ et al (2015) Sessile serrated polyp prevalence determined by a colonoscopist with a high lesion detection rate and an experienced pathologist. Gastrointest Endosc 81:517-524

5. Gurudu SR, Heigh RI, De Petris G et al (2010) Sessile serrated adenomas: demographic, endoscopic and pathological characteristics. World J Gastroenterol 16:3402-3405

6. Spring KJ, Zhao ZZ, Karamatic R et al (2006) High prevalence of sessile serrated adenomas with BRAF mutations: a prospective study of patients undergoing colonoscopy. Gastroenterology 131: $1400-1407$

7. Carr NJ, Mahajan H, Tan KL et al (2009) Serrated and non-serrated polyps of the colorectum: their prevalence in an unselected case series and correlation of BRAF mutation analysis with the diagnosis of sessile serrated adenoma. J Clin Pathol 62:516-518

8. Neumann J, Reu S, Kirchner T (2012) Prognostic marker profiles for risk of distant metastases in colorectal cancer. Pathologe 33:3944

9. Kahi CJ, Li X, Eckert GJ et al (2012) High colonoscopic prevalence of proximal colon serrated polyps in average-risk men and women. Gastrointest Endosc 75:515-520

10. Hetzel JT, Huang CS, Coukos JA et al (2010) Variation in the detection of serrated polyps in an average risk colorectal cancer screening cohort. Am J Gastroenterol 105:2656-2664

11. Khalid O, Radaideh S, Cummings OW et al (2009) Reinterpretation of histology of proximal colon polyps called hyperplastic in 2001 . World J Gastroenterol 15:3767-3770

12. Singh H, Bay D, Ip S et al (2012) Pathological reassessment of hyperplastic colon polyps in a city-wide pathology practice: implications for polyp surveillance recommendations. Gastrointest Endosc 76:1003-1008

13. Wong NA, Hunt LP, Novelli MR et al (2009) Observer agreement in the diagnosis of serrated polyps of the large bowel. Histopathology 55:63-66

14. Ensari A, Bilezikci B, Carneiro F et al (2012) Serrated polyps of the colon: how reproducible is their classification? Virchows Arch 461: 495-504
15. Aust DE, Baretton GB (2010) Serrated polyps of the colon and rectum (hyperplastic polyps, sessile serrated adenomas, traditional serrated adenomas, and mixed polyps)-proposal for diagnostic criteria. Virchows Arch 457:291-297

16. Lieberman DA, Rex DK, Winawer SJ et al (2012) Guidelines for colonoscopy surveillance after screening and polypectomy: a consensus update by the US multi-society task force on colorectal cancer. Gastroenterology 143:844-857

17. Pox CP, Schmiegel W (2013) German S3-guideline colorectal carcinoma. Dtsch Med Wochenschr 138:2545

18. Schachschal G, Mayr M, Treszl A et al (2014) Endoscopic versus histological characterisation of polyps during screening colonoscopy. Gut 63:458-465

19. Larsen K, Merlo J (2005) Appropriate assessment of neighborhood effects on individual health: integrating random and fixed effects in multilevel logistic regression. Am J Epidemiol 161:81-88

20. Gao Q, Tsoi KK, Hirai HW, et al (2015) Serrated polyps and the risk of synchronous colorectal advanced neoplasia: a systematic review and meta-analysis. Am J Gastroenterol

21. Bettington M, Walker N, Clouston A et al (2013) The serrated pathway to colorectal carcinoma: current concepts and challenges. Histopathology 62:367-386

22. Leggett B, Whitehall V (2010) Role of the serrated pathway in colorectal cancer pathogenesis. Gastroenterology 138:2088-2100

23. Kriegl L, Vieth M, Kirchner T et al (2012) Up-regulation of c-MYC and SIRT1 expression correlates with malignant transformation in the serrated route to colorectal cancer. Oncotarget 3:1182-1193

24. Torlakovic E, Skovlund E, Snover DC et al (2003) Morphologic reappraisal of serrated colorectal polyps. Am J Surg Pathol 27:6581

25. Torlakovic E, Snover DC (1996) Serrated adenomatous polyposis in humans. Gastroenterology 110:748-755

26. Anders M, Bahr C, El-Masry MA et al (2014) Long-term recurrence of neoplasia and Barrett's epithelium after complete endoscopic resection. Gut 63:1535-1543

27. Bettington M, Walker N, Rosty C et al (2014) Critical appraisal of the diagnosis of the sessile serrated adenoma. Am J Surg Pathol 38: 158-166 